\title{
An extension of the multi-output state-contingent production model
}

\author{
Walter Briec • Laurent Cavaignac
}

Published online: 3 January 2008

(C) Springer-Verlag 2007

\section{Erratum to: Economic Theory DOI 10.1007/s00199-007-0308-6}

The original version of this article unfortunately contained a few typographical mistakes. The presentation of Equation 8, Example 2.1, and 2.2 should be corrected. The corrected versions are given below.

Equation 8

$$
\|\tilde{y}\|_{L_{\varphi}^{2}}=\left(\int_{\Omega} \varphi\|\tilde{y}\|^{2} d \mathbb{P}\right)^{1 / 2}=\left(\mathbb{E}_{\varphi}\left[\|\tilde{y}\|^{2}\right]\right)^{1 / 2}
$$

Example 2.1

$$
\|\tilde{y}\|_{L^{2}}=\left(\int_{\Omega}\|\tilde{y}\|^{2} d \mathbb{P}\right)^{1 / 2}=\left(\sum_{n \in N}\|\tilde{y}(n)\|^{2} \mathbb{P}(n)\right)^{1 / 2} .
$$

The online version of the original article can be found under doi:10.1007/s00199-007-0308-6.

W. Briec

LAMPS, University of Perpignan, Via Domitia, 52 Avenue Villeneuve, 66860 Perpignan Cedex, France

L. Cavaignac $(\varangle)$

GEREM, University of Perpignan, Via Domitia, 52 Avenue Villeneuve, 66860 Perpignan Cedex, France

e-mail: laurent.cavaignac@univ-perp.fr 


\section{Example 2.2}

$$
\|\tilde{y}\|_{L^{2}}=\left(\int_{\Omega}\|\tilde{y}\|^{2} d \mathbb{P}\right)^{1 / 2}=\left(\sum_{s-1}^{S}\|\tilde{y}(s)\|^{2} \mathbb{P}(s)\right)^{1 / 2} .
$$

